\title{
Cervical Spinal Cord Neoplasm in a Patient with an Implanted Cervical Spinal Cord Stimulator: The Controversial Role of MAgnetic Resonance Imaging
}

\author{
Rinoo V. Shah MD, Harold K. Smith, MD, Joanne Chung, MD, Amr Hegazi MD, and Gabor B. Racz, MD
}

Objective: To discuss the diagnostic relevance and safety concerns of performing magnetic resonance imaging (MRI) in the presence of an implanted spinal cord stimulator (SCS).

Case Report: A 39-year old man with CRPS I and major depression fell and fractured his humerus. A cervical SCS had been placed several years earlier. After the fall, he developed progressive lower extremity weakness. Eventually, he became wheelchair dependent and required assistance with transfers. These symptoms were attributed to CRPS and deconditioning. He was admitted to an inpatient rehabilitation unit for functional restoration. At presentation, he reported developing neck and upper thoracic pain, which started several months before the fall-a pain that was distinct from his total body pain. A contrast-enhanced CT scan of the neck was normal, except for the spinal cord stimulator induced artifact. Our pain service was consulted. After performing a neurological exam, cervical myelopathy was suspected. An MRI of the spine demonstrated a mass compressing the cervical spinal cord. The patient tolerated the MRI, despite the presence of a neurostimulator. The mass was identified as an extramedullary, intradural schwannoma and was completely excised during surgery. The patient made a profound neurological recovery.

Conclusion: The diagnostic value of MRI may outweigh the potential dangers of using this imaging modality when a patient with a neurostimulation device presents with a new-onset neurological deficit. Thorough informed consent and close physician monitoring of these patients during the MRI is imperative.

Keywords: Spinal cord stimulation; neuromodulation; neurostimulation; magnetic resonance imaging, safety; magnetic resonance imaging, implants; magnetic resonance imaging, heating; MRI
Magnetic resonance imaging (MRI) studies should not be performed in patients with an implanted spinal cord stimulator, according to the two largest manufacturers of these neurostimulation devices: Medtronic ${ }^{\circledR}(1)$ and Advanced Neuromodulation Systems ${ }^{\circledR}$ (2). Due to these warnings, many imaging centers will not

From International Pain Institute, Department of Anesthesiology and Pain Services, Texas Tech University Health Sciences Center, Lubbock, Texas, Lubbock Neurosurgical Associates, Lubbock, Texas, and Department of Radiology, University Medical Center, Lubbock, Texas. Address Correspondence: Rinoo V. Shah, MD, Assistant Professor, International Pain Institute, 4430 South Loop 289, Lubbock, Texas 79414

E-mail: rinoo shah@yahoo.com

Suppliers: Itrel $\| \circledast$, Itrel $I I \circledast$, SynergyTM, and Pisces Quad-Plus ${ }^{\circledR}$ are all registered trademarks of Medtronic World Headquarters, 710 Medtronic Parkway, Minneapolis, MN 554325604, Phone: (763) $514-4000$ or $574-4000$ GenesisTM and Genesis XPTM are registered trademarks of Advanced Neuromodulation Systems, 6501 Windcrest Drive, Suite 100, Plano, Texas 75024 Ph: 972-309-8000

Support: Dr. Gabor B. Racz is a consultant to Medtronic Inc. However, no external funding was received for this study and no financial benefit will be conferred on the authors or on the device manufacturers mentioned herein

Conflict of Interest: None perform MRIs on patients with implanted neurostimulation devices.

However, certain emergent clinical scenarios require MR imaging for diagnosis-MRIs have a high sensitivity in detecting soft tissue abnormalities of the spine, such as infection (3), spinal cord trauma (4-7), bleeding (8), and neoplasm (9). In fact, MR imaging is the gold standard for the diagnosis of soft tissue spinal tumors, particularly if there is a neurological deficit (9).

Neoplasms should be routinely considered in the differential diagnosis of spinal pain and neurological impairment, but they may be overlooked in pain clinics: the prevalence of spinal tumors in primary care and multidisciplinary spine clinics is less than $1 \%$ (10). Apprehension about performing MRIs in the presence of neurostimulation devices and reduced clinical vigilance may lead to failure or delay in diagnosing spinal tumors in this patient population.

We present the first case of a neoplasm that developed within the area of the spinal cord undergoing neurostimulation a case in which MR imaging was essential for diagnosis and treatment planning.

\section{Case Description}

A 39 year-old male fractured his left humerus after falling from a wheelchair. His past medical history was notable for CRPS I and major depression. CRPS I developed in his left lower extremity 5 years earlier and then spread to the remaining limbs. Pain management consisted of physical therapy, analgesics, psychological counseling, and neuromodulation: cervical and thoracic spinal cord stimulators (SCS) and an intrathecal infusion pump.

His total body pain, and particularly the neck pain, worsened after the fall. He began feeling weak and numb in his lower extremities and trunk within a few days. One month post-injury, he was only able to stand and pivot. Two months postinjury, he became wheelchair bound and required assistance with transfers. He became more depressed, despite increases in his antidepressant regimen. Due to the progression of these symptoms and limitations in self-care, the patient's primary care physician arranged a transfer to our hospital's inpatient rehabilitation facility. The goal was to restore function.

On admission to the rehabilitation service, a CT scan of the neck with and 
without contrast was obtained. The stimulator leads were in the cervical epidural space, but they had migrated ventro-laterally from the dorsal midline. The leads produced a significant amount of scatter in the spinal canal and this obscured image interpretation. Both the interpreting radiologist and our co-author (JC) did not identify any pathology on the CT scan, even with contrast enhancement.

Our pain service was consulted to provide ongoing analgesic recommendations. The patient again reported an increase in neck and upper thoracic pain, but distinctly felt this pain developed several months before the fall. In fact, during this time period, analgesics were escalated and his cervical spinal cord stimulator was reprogrammed several times. The patient stopped using his cervical stimulator, due to lack of relief. This pain prevented him from lying supine for any length of time.

The patient was visibly anxious and distressed. Both lower extremities were swollen. The left arm was in a sling. Both arms demonstrated areas of hyperesthesia

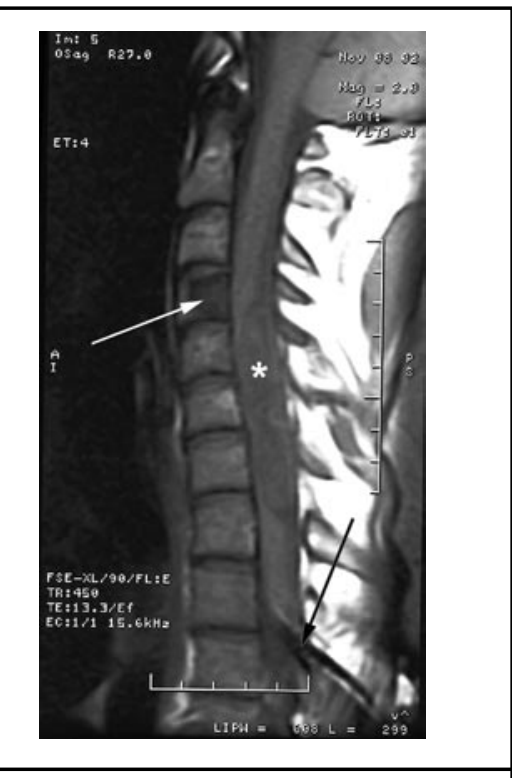

Fig. 1. Mid-sagittal, T1-weighted, FSE image of the cervical spine: heterogeneous, isotense lesion in the spinal canal, extending from the C4 to the T1 level(*); low signal lesion in the C4 vertebral body (white arrow); spinal cord stimulator leads with both high and low signal entering upper thoracic epidural space (black arrow). and allodynia. There was mild neck stiffness but Lhermitte's sign was absent. Both light touch and pinprick were reduced below the T5 dermatome; pinprick was specifically absent over the thighs and legs. There was virtually no motor strength in both lower extremities. The upper extremities demonstrated mild weakness distally, in the hands. The toes had an extensor response to Babinski testing. There was sustained ankle clonus, bilaterally. Lower extremity tone was significantly increased. Hoffmann's sign was absent and upper extremity reflexes were within normal limits. The physical examination was consistent with a cervical or upper thoracic myelopathy. Laboratory studies were normal with the exception of an elevated C-reactive protein.

We decided to obtain an MRI of the entire spine, with and without contrast, on an emergent basis. The patient and his wife were counseled at length about the risks of obtaining an MRI including electrode heating, nerve damage, paralysis, lead migration, equipment failure, and

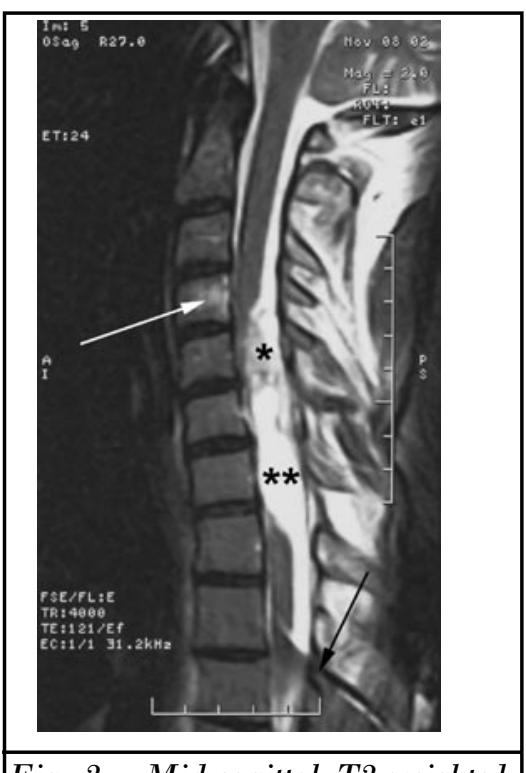

Fig. 2. Mid-sagittal T2-weighted FSE of the cervical spine: hyperintense lesion in the spinal canal, extending from the C4 to the TI level; the upper portion of lesion is heterogeneous (*) and lower portion is homogeneous (**); high signal lesion in the C4 vertebral body (white arrow); leads with both high and low signal entering the upper thoracic epidural space (black arrow). pain. We explained that a physician would be in the MRI suite with the patient at all times and would repeatedly query the patient about his ability to tolerate the study. The patient agreed to the MRI and signed a witnessed informed consent.

The internal programmable generator (IPG) only permitted bipolar lead combinations. The voltage was set to zero and the IPG was turned off. Our top priority was to obtain an image of the entire spine in the limited time available: the patient was uncomfortable lying in the supine position. To maintain constant verbal contact with the patient, the study could not be performed with intravenous sedation or general anesthesia. We requested contrast enhancement but the patient could not tolerate lying supine and this was not given.

The cervical spine MRI was performed with a sagittal T1-weighted fast spin echo (FSE), sagittal T2-weighted FSE, axial T2-weighted FSE, and axial T1-weighted spin echo images. The MRI demonstrated a large, lobular, relatively well circumscribed mass extending from C4-5 to T2 that was compressing and displacing the cord ventrally and rightwards (Figures 1-4). The greatest degree of spinal cord compression was located close to C7-T1, which may have accounted for the relative neurological sparing of the upper extremities.

The lesion was isointense on T1weighted images (Fig. 1), hyperintense on T2-weighted images (Figs. 2-4), cystic in composition and appeared benign. The images were consistent with an extramedullary, intradural mass.

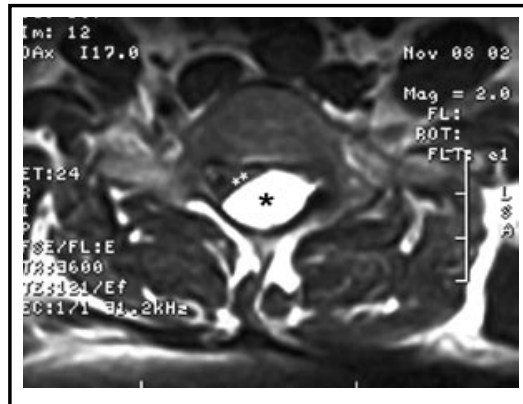

Fig. 3. Axial T2-weighted FSE image at the level of the midportion of the C7 vertebral body: hyperintense lesion (*) severely compressing the spinal cord (**) and displacing it ventrolaterally to the right. 


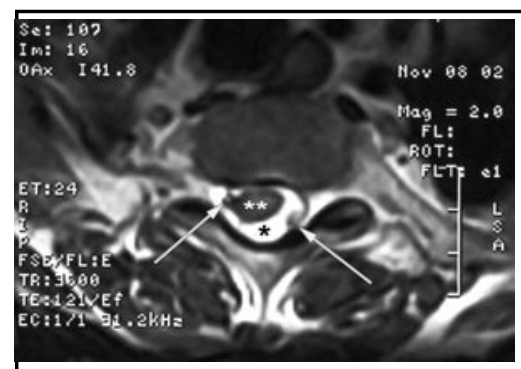

Fig. 4. Axial T2-weighted FSE image at the level of the T1-2 intervertebral disc: percutaneously placed spinal cord stimulation leads with low signal intensity and surrounded by hyperintense artifact; the leads do not distort the image (white arrows); again note the lesion (*) displacing the spinal cord (**) ventrolaterally to the right.

Cervical stimulator leads could be identified entering the epidural space at T1-T2 (Figs. 1, 2, and 4). These leads did not cause significant artifact, but were of a low signal intensity that was surrounded by a higher signal intensity (Figs. 1,2 and 4). In the posterior C4 vertebral body, the signal intensity was abnormal. This nonspecific finding was of low signal on $\mathrm{T} 1$ images (Figure 1) and a less pronounced, but higher signal on T2 images (Fig. 2).

The patient was placed immediately on an intravenous steroid protocol. The next day he underwent a C4-T1 laminectomy and excision of an intradural and extramedullary mass. The mass severely compressed the cord to the right. The mass was soft, lobulated, grayish in color, and measured $3.5 \times 2.5 \times 1$ cubic centimeters (Fig. 5). The mass showed a pattern characteristic of a schwannoma. The mass was well encapsulated with dense Antoni $\mathrm{A}$ and looser Antoni B areas. There was no evidence of infection at the time of surgery. Additionally, both the pre and postoperative CT scans failed to show any evidence of osteolysis or osteomyelitis.

The neurosurgeon did not see the leads in the operative field. This may have been because they migrated ventrolaterally and the surgical approach was posterior by laminectomy. Additionally, the tumor was encapsulated and there was no evidence of infection, so the SCS electrode and lead were not removed.

The neurological exam 24 hours following surgery significantly improved. The patient's sensation to light touch and

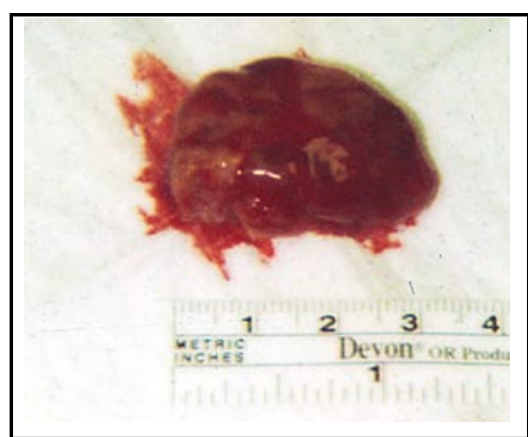

Fig. 5. Gross specimen of excised intradural extramedullary, cervical schwannoma

pinprick in all lower extremity and truncal dermatomes improved. Lower extremity myotomal strength improved to 4 to $4+/ 5$. The lower extremities were less hyperreflexic. Clonus decreased to 10 beats on each foot. At follow-up one year later, the patient demonstrated a profound recovery. He could walk without assistance about 300 feet and was actively participating in pool therapy. His motor exam was completely normal. His sensory exam only demonstrated mild patchy sensory loss on the right upper thigh. The left foot still had 9-10 beats of clonus, but none on the right. Rectal tone and urinary continence were preserved throughout his clinical course.

Post-operatively, the patient's pain in the upper thoracic spine abated, but the total body pain persisted, which was controlled with the intrathecal pump and oral analgesics. Note should be made that the SynchroMed pump (Medtronic Neurological, Minneapolis, Minnesota) can be safely scanned by MRI; the pump was not a concern during the scanning (see Medtronic website: www.medtronic.com).

There is a possibility that the schwannoma may have contributed to the total body pain, in which case we would have to reclassify the etiology of his pain as a central pain syndrome emanating from spinal cord injury. However, the development of the CRPS predated any suspicion of a tumor and developed following a musculoskeletal injury almost 5 years earlier. The increase in upper thoracic pain, in retrospect, was the only harbinger of the tumor, in terms of pain symptomatology. Also, the total body pain did not significantly improve after tumor removal.

\section{DISCUSSION}

Despite controversy, MRIs are routinely performed on patients with SCS devices $(11,12)$. Safety guidelines have been developed for deep brain stimulation (DBS) devices, based on in vitro and in vivo safety studies $(12,13)$. Despite similarities in lead design between DBS and SCS systems, the DBS guidelines cannot be applied to SCS systems: published neurostimulation safety guidelines must only apply to leads, lead/battery combinations, and MR imaging protocols that are tested $(12,13)$.

Several mechanisms impact the safety of performing an MRI in the presence of a neurostimulation device. The static magnetic field can affect the extension cable or the IPG, but not the electrode contacts-these platinum and iridium contacts are not ferromagnetic (12). MR image acquisition depends on the delivery of a pulsed radiofrequency field (RF) that generates voltage. Voltage induces current in the extension wires and the leads; some of this current passes through the electrode contact points into surrounding tissues (14). This current can depolarize neural tissue and generate heat secondary to friction losses (12).

Adverse events associated with MR imaging include lead movement, lead dislodgement, and neural tissue activation (14), but excessive heating induced by RF field effects on the stimulator leads during imaging is the most worrisome. Irreversible neural tissue damage occurs if temperatures exceed $45^{\circ} \mathrm{C}(14)$. Factors associated with DBS electrode heating include electrode configuration, wire impedance and breakage, the type of radiofrequency coil, and the MRI-specific absorption rate (SAR) $(13,14)$. For instance, a 1.5 Tesla MRI and body RF transmit coil can lead to an in-vitro temperature increase that varies from $2.5-25.3^{\circ} \mathrm{C}$, when testing DBS leads (13).

The SAR is a measure of the amount of radiofrequency energy absorbed by the body and is measured in Watts $/ \mathrm{kg}$. For MRIs, the Food and Drug Administration suggests that the SAR should not exceed 4 Watts/kg over 15 minutes averaged for the whole body, 3 Watts/kg over 10 minutes averaged for the head, 8 Watts/ $\mathrm{kg}$ over 5 minutes per gram of tissue for the head and torso, or 12 Watts/kg over 5 minutes per gram of tissue for the extremities (15). 
There is a linear correlation between the temperature increase around the DBS electrode and the SAR. If the upper SAR limit is set to 2.4 Watts $/ \mathrm{kg}$ for the head and 0.9 Watts/kg averaged for the whole body, then a temperature increase of $2.0^{\circ} \mathrm{C}$ occurs with DBS leads and this increase is clinically tolerated (14). The relationship between the SAR and temperature increases in SCS systems has not been studied and a linear relationship should not be assumed (13). The SAR values during the MR study on our patient were not recorded by the MR technologist, but SAR limits had already been programmed into MR sequences used.

Tronnier et al (12) rigorously evaluated the in vitro and in vivo safety of MR imaging on patients with neurostimulation devices. Their findings were: (1) an MRI that is performed on an area that is removed from the neurostimulation device is safe; (2) MR imaging can be performed on deep brain leads connected to an IPG, whether the latter structure is on or off; (3) an MRI is possible with percutaneous spinal cord stimulation leads; (4) further investigations using larger plate electrodes must be performed; (5) the tested IPGs, ITREL II ${ }^{\circledR}$ and III ${ }^{\circledR}$, caused no discomfort or injury due to mechanical forces, heat, or induced current, when coupled to DBS leads; (6) the tested IPGs experienced no changes in parameter settings, but were automatically switched on or off.

Overall, these recommendations (12) are for IPGs that allow only single channel programming and both mono- or bipolar settings: ITREL II and III. Only one patient in Tronnier et al's (12) group had a percutaneously placed spinal cord stimulation lead (Pisces QuadPlus ${ }^{\circledR}$ ) and this was connected to an untested IPG: Synergy $^{\mathrm{TM}}$. A Synergy ${ }^{\mathrm{TM}}$ IPG permits single or dual channel programming and only bipolar settings. Our patient had two QuadPlus $^{\circledR}$ leads and a Synergy ${ }^{\mathrm{TM}}$ IPG. In Tronnier et al's (12) group, four patients had plate electrodes. These electrodes are implanted via a laminotomy into the spinal epidural space. Two out of these four patients experienced brief, but significant dysesthesias.

The findings and recommendations of Tronnier et al (12) are only valid for the neurostimulation devices tested, 0.2 or 1.5 Tesla MRIs, the specific MR sequences used, and the specific lead/IPG combinations. Newer IPGs, low impedance exten- sions, peripheral nerve electrode arrays, and neurostimulation devices produced by other manufacturers have not been tested. Thus, the author's (12) recommendations, for all practical purposes cannot be generalized to the majority of spinal cord system implants in use today.

Tronnier et al (12) advise that a physician be present during the MRI. This physician should be familiar with the neurostimulation device. If the patient complains of any uncomfortable sensation, the study should be stopped. We adhered to this protocol: the IPG was turned off, voltage was set to zero, settings were bipolar, and a physician was present next to the patient during the MRI. The patient was repeatedly queried about his symptoms and he described none. Tronnier et al's (12) study also demonstrated that the induced voltage differences between electrode contacts are reduced if the electrode remains connected to the IPG, rather than disconnected. This is why we did not surgically remove the IPG prior to the MR study.

Several other case reports have demonstrated $(11,16,17)$ that an MRI can be safely performed in patients with SCS devices. Kirakopoulos et al (11) safely performed functional MRI studies of the brain using a conventional 1.5-Tesla magnetic resonance system on three patients undergoing a trial with percutaneously placed spinal cord stimulation electrodes at the T11-12 level. Long trial leads were used and the leads were taped separately from one another to prevent electrical arcing. Parisod et al (16) obtained an MRI of the brain and cervical spine on a patient with an implanted cervical spinal cord stimulator with a $2 \times 4$ electrode array. The IPG was turned off. The patient had no adverse sequelae. The device manufacturer and type of implant (percutaneous versus laminotomy) was not specified and there was no comment as to whether the patient was accompanied by a physician during the study. Liem et al (17) performed an MRI on three patients with spinal cord stimulators and all tolerated the procedure safely. In spite of these reports, practitioners may rely on imaging modalities that are less sensitive than an MRI in patients with neurostimulation devices, due to concerns of causing iatrogenic injury.

Arguably, one could have considered performing a CT-myelogram in our patient. CT-myelography may be preferred to MRI during the pre-operative evaluation of myelopathy in some circumstances. Both imaging modalities provide unique and complementary information in cervical spondylotic myelopathy (18). However, there were several concerns. The absence of significant cervical spondylosis on the initial CT scan, the presence of SCS lead-induced artifact on the CT scan, the younger age of the patient, and the risks of performing a lateral C1-2 puncture for contrast instillation argued against using a CT-myelogram as the initial study. Furthermore, MRI is the preferred method for evaluating myelopathy secondary to intrinsic cord pathology. MRIs have superior soft tissue contrast definition, capable of defining spinal cord edema, hemorrhage, and atrophy (18). MRIs are noninvasive and intravenous contrast instillation can demonstrate cord enhancement in the patient with myelopathy (18). MRI has become the preferred screening method for the evaluation of cervical spine myelopathy. CT-myelography may be considered and provide valuable additional information if the MRI is ambiguous or technically suboptimal (18).

The MRI demonstrated a mass causing significant extrinsic cord compression. The electrodes did not alter the image quality. In retrospect, intravenous contrast would have been helpful, but we were concerned about finishing the study - the patient was uncomfortable lying on the MR table. After seeing the mass in the cervical spine on the first few images, we thought it would be more prudent to use the limited time to obtain images of the entire spine. In the presence of a spinal tumor, imaging the entire spine is prudent due to the risk of seeding (19). If there were any doubts about the diagnosis, a CT-myelogram could have been obtained. However, our neurosurgical colleagues considered the diagnostic information on the MRI to be sufficient to proceed with surgery.

The MR sequences used on our patient, with the exception of not administering intravenous contrast, were consistent with those that are recommended for the evaluation of spinal tumors: sagittal T1-weighted spin-echo, sagittal T2weighted fast spin-echo, and corresponding axial images through the area of interest (19).

Intradural spinal tumors are onetenth as common as intracranial tumors and fortunately, most are benign (20). 
Spinal extramedullary, intradural tumors account for $2 / 3$ of all intraspinal neoplasms $(20,21)$. The mean duration of symptoms prior to diagnosis is approximately thirty months. At the cervical level, only $33 \%$ of these patients present with pain and $43 \%$ present with motor weakness (20).

Schwannomas are typically intradural-extramedullary and solitary, unless they occur in the presence of neurofibromatosis-2 (19). Schwannomas or nerve sheath tumors are uncommon (21) and are even more uncommon in the cervical spine. Schwannomas typically afflict both sexes with an equal predilection and the average age at presentation ranges from 39-43 years old. On an MRI, Schwannomas are fairly isointense on T1-weighted images and usually bright on T2-weighted images. The Schwannoma in our patient met all these criteria. If contrast had been used, the tumor likely would have enhanced on T1-weighted images (19).

A complete excision of the schwannoma can usually be achieved and this correlates with good post-operative outcomes (21). The degree of pre-operative neurological dysfunction and length of clinical history adversely affect outcome. Despite the length of our patient's clinical history and poor functional status, he did well with complete excision (21).

A multilevel, posterior approach, decompressive laminectomy and single stage tumor removal is the most common procedure (20). Complete excision is successful in the majority of patients and is advocated. This posterior approach was used in our patient. The tumor was intradural and extramedullary and easily excised. With other presentations, different surgical strategies may have to be performed depending on the tumor location and extension: anterior and lateral approaches, staged procedures, and stabilization. Reported surgical mortality and morbidity rates are $0 \%$ and less than $15 \%$, respectively (20). Pain improves in over $90 \%$ of patients and in the cervical region, neurological recovery approaches $60 \%$ (20).

Pathologically, schwannomas originate from Schwann cells, which produce myelin (19). These tumors often originate from nerve roots, particularly the posteriorly located sensory roots. These tumors are well encapsulated and push, rather than invade, the nerve root (19). Histologically, they are composed of dense bundles of spindle cells (Antoni A fi- bers), which are not present in meningiomas (19). Less compact (Antoni B fibers) spindle cells and cystic degeneration are also common. Meningiomas and neurofibromas do not have cystic characteristics (19). Our patient's tumor histology was characteristic of a schwannoma.

Apart from the cervical location of his tumor, our patient represents the 'classic' patient with an intradural, extramedullary schwannoma, including a delay in diagnosis, symptom complex, age, imaging findings, surgical outcome, and postoperative functional outcomes.

\section{Conclusion}

Spinal cord stimulation systems have not received an MRI-safe designation by the Food and Drug Administration (22). The greatest hazard is the risk of heating and permanently destroying vital neural tissue, due to RF field effects on the stimulator leads. Under certain emergent circumstances, an MRI is essential for diagnosis and for planning therapy. If an MRI is imperative, a physician familiar with the neurostimulation device should monitor the patient closely, the internal programmable generator should be set to off, the electrode contacts should be in a bipolar mode, the MR specific absorption rate should be tightly controlled, and a thorough informed consent should be obtained.

\section{REFERENCES}

1. Magnetic Resonance Imaging. Consideration for Patients with a Medtronic $\circledR$ Neurostimulation System for Chronic Pain. In: Patient Services and Technical Services Standard Recommendations Rev. 1. Medtronic $\circledast$ Incorporated, April 2, 2002.

2. Indications for Use, Contraindications, and Warnings. In: Genesis ${ }^{\top \mathrm{M}}$ and Genesis XP'M Clinicians Manual, Advanced Neuromodulation Systems ${ }^{\circledR}$, October 2001.

3. Silber JS, Anderson DG, Vaccaro AR et al. Management of postprocedural discitis. Spine J 2002; 2:279-287.

4. Mascalchi M, Dal Pozzo G, Dini C et al. Acute spinal trauma: prognostic value of MRI appearances at 0.5 T. Clin Radiol 1993; 48:100-108.

5. Curati WL, Kingsley DP, Kendall BE et al. MRI in chronic spinal cord trauma. Neuroradiology 1992; 35:30-35.

6. Benzel EC, Hart BL, Ball PA et al. Magnetic resonance imaging for the evaluation of patients with occult cervical spine injury. I Neurosurg 1996; 85:824-829.

7. Radiographic Assessment of the Cervical Spine in Symptomatic Trauma Patients. Neurosurgery 2002; 50:S36-S43.

\section{Author Affiliation:}

Rinoo V. Shah, MD

Assistant Professor

Department of Anesthesiology and

Pain Services

International Pain Institute

Texas Tech University Health Sciences

Center

4430 South Loop 289

Lubbock, Texas 79414

E-mail: rinoo_shah@yahoo.com

Harold K. Smith, MD

Neurosurgeon

Lubbock Neurosurgical Associates

3506 21st St \#401

Lubbock, TX 79410

Joanne Chung, MD

Department of Radiology

University Medical Center

PO Box 5980

Lubbock, TX 79408-5980.

\section{Amr Hegazi, MD}

International Pain Institute

Department of Anesthesiology and

Pain Services

Texas Tech University Health

Sciences Center

4430 South Loop 289

Lubbock, Texas 79414

Gabor B. Racz, MD

Grover E. Murray Professor and

Chairman Emeritus

International Pain Institute

Texas Tech University Health Sciences

Center

36014 th Street

Lubbock, Texas 79430

E-mail: paula.brashear@ttuhsc.edu

8. Young PC, Petersilge CA. MR imaging of the traumatized lumbar spine. Magn Reson Imaging Clin N Am 1999; 7:589-602.

9. Parikh SN, Crawford AH. Orthopaedic implications in the management of pediatric vertebral and spinal cord tumors: A retrospective review. Spine 2003; 28:23902396.

10. Slipman CW, Patel RK, Botwin K et al. Epidemiology of spine tumors presenting to musculoskeletal physiatrists. Arch Phys Med Rehabil 2003; 84:492-495.

11. Kiriakopoulos ET, Tasker RR, Nicosia S et al. Functional magnetic resonance imaging: a potential tool for the evaluation of spinal cord stimulation: Technical case report. Neurosurgery 1997; 41:501-504.

12. Tronnier VM, Staubert A, Hahnel S et al. Magnetic resonance imaging with implanted neurostimulators: An in vitro and in vivo 
study. Neurosurgery 1999; 44:118-126.

13. Sharan A, Rezai AR, Nyenhuis JA et al. MR safety in patients with implanted deep brain stimulation systems (DBS). Acta Neurochir Suppl 2003; 87:141-145.

14. Finelli DA, Rezai AR, Ruggieri PM et al. $M R$ imaging-related heating of deep brain stimulation electrodes: in vitro study. AJNR Am J Neuroradiol 2002; 23:17951802.

15. Zaremba L. Guidance for Industry and FDA Staff Criteria for Significant Risk Investigations of Magnetic Resonance Diagnostic Devices: Office of Device Evalu ation, Center for Devices and Radiological Health, Food and Drug Administration, 07/14/2003 (accessed February 4, 2004 at http://www.fda.gov/cdrh/ode/guidance/ 793.pdf)

16. Parisod E, Murray RF, Cousins MJ. Conver sion disorder after implant of a spinal cord stimulator in a patient with a complex regional pain syndrome. Anesth Analg 2003; 96:201-206.

17. Liem LA, van Dongen VC. Magnetic resonance imaging and spinal cord stimulation systems. Pain 1997; 70:95-97.

18. Shafaie FF, Wippold FJ, Gado M et al. Comparison of computed tomography myelography and magnetic resonance imaging in the evaluation of cervical spondylotic myelopathy and radiculopathy. Spine 1999; 24:1781-1785.

19. Lee RR. MR imaging of intradural tumors of the cervical spine. Magn Reson Imaging
Clin N Am 2000; 8:529-540.

20. el-Mahdy W, Kane PJ, Powell MP et al. Spinal intradural tumors: Part I--Extramedullary. Br J Neurosurg 1999; 13:550-557.

21. Albanese V, Platania N. Spinal intradural extramedullary tumors. Personal experi ence. J Neurosurg Sci 2002; 46:18-24.

22. CDRH Magnetic Resonance Working Group Draft Document: A Primer on Medical Device Interactions with Magnetic Resonance Imaging Systems. Rockville, Md.: Office of Science and Technology, Center for Devices and Radiological Health, Food and Drug Administration, 1997 (accessed February 7, 2004 at http://www.fda.gov/ cdrh/ode/primerf6.html) 